\title{
Naltrexone Maintenance Decreases Cannabis Self-Administration and Subjective Effects in Daily Cannabis Smokers
}

\author{
Margaret Haney*, , Divya Ramesh ${ }^{1,2}$, Andrew Glass ${ }^{1,3}$, Martina Pavlicova ${ }^{1,4}$, Gillinder Bedi' and \\ Ziva D Cooper
}

'Division on Substance Abuse, New York State Psychiatric Institute and Department of Psychiatry, Columbia University Medical Center, New York, NY, USA; ${ }^{2}$ Division of Addiction Psychiatry, Virginia Commonwealth University, Richmond, VA, USA; ${ }^{3}$ Division of Biostatistics, Department of Psychiatry, New York State Psychiatric Institute, New York, NY, USA; ${ }^{4}$ Department of Biostatistics, Mailman School of Public Health, Columbia University, New York, NY, USA

\begin{abstract}
Given that cannabis use is increasing in the United States, pharmacological treatment options to treat cannabis use disorder are needed. Opioid antagonists modulate cannabinoid effects and may offer a potential approach to reducing cannabis use. In this double-blind, placebo-controlled human laboratory study, we assessed the effects of naltrexone maintenance on the reinforcing, subjective, psychomotor, and cardiovascular effects of active and inactive cannabis. Nontreatment-seeking, daily cannabis smokers were randomized to receive naltrexone ( $50 \mathrm{mg}: n=18 \mathrm{M}$ and $5 \mathrm{~F}$ ) or placebo $(0 \mathrm{mg} ; n=26 \mathrm{M}$ and $2 \mathrm{~F}$ ) capsules for 16 days. Before, during, and after medication maintenance, participants completed 10 laboratory sessions over 4-6 weeks, assessing cannabis' behavioral and cardiovascular effects. Medication compliance was verified by observed capsule administration, plasma naltrexone, and urinary riboflavin. Relative to placebo, maintenance on naltrexone significantly reduced both active cannabis self-administration and its positive subjective effects ('good effect'). Participants in the placebo group had 7.6 times (95\% Cl: I.I-5 I.8) the odds of self-administering active cannabis compared with the naltrexone group. This attenuation of reinforcing and positive subjective effects also influenced cannabis use in the natural ecology. Naltrexone had intrinsic effects: decreasing ratings of friendliness, food intake, and systolic blood pressure, and increasing spontaneous reports of stomach upset and headache, yet dropout rates were comparable between groups. In summary, we show for the first time that maintenance on naltrexone decreased cannabis self-administration and ratings of 'good effect' in nontreatment-seeking daily cannabis smokers. Clinical studies in patients motivated to reduce their cannabis use are warranted to evaluate naltrexone's efficacy as a treatment for cannabis use disorder.

Neuropsychopharmacology (20I5) 40, 2489-2498; doi:I0.I038/npp.20I5.108; published online 20 May 2015
\end{abstract}

\section{INTRODUCTION}

Cannabis use in the United States is increasing (Substance Abuse and Mental Health Services Administration (SAMHSA), 2013) and epidemiological data suggest that rates may rise further with cannabis legalization (Cerdá $\mathrm{et} \mathrm{al}$, 2012). Currently, $\sim 24 \%$ of patients entering treatment for substance abuse have a diagnosis of cannabis use disorder (CUD; SAMHSA, 2012), yet few patients are able to achieve continued abstinence (Marijuana Treatment Project Research Group, 2004; Budney et al, 2006; Kadden et al, 2007; Levin et al, 2011; Weinstein et al, 2014). There is thus a clear and pressing need to improve treatment

\footnotetext{
*Correspondence: Dr M Haney, Division on Substance Abuse, Columbia University Medical Center, New York State Psychiatric Institute, I05 I Riverside Drive, Unit 120, New York, NY I0032, USA, Tel: + I 646774 6I53, Fax: + I 646774 6I4I,

E-mail: mh235@columbia.edu

Received 17 November 2014; revised 9 April 2015; accepted 10 April 2015; accepted article preview online 16 April 2015
}

outcomes for cannabis use disorder, and pharmacological options are one important strategy needing development.

The first step in developing potential treatment medications for substance use disorders is to define which feature of problematic drug use to target. For example, medications can target relapse by minimizing the factors that increase the likelihood that an abstinent cannabis smoker will return to cannabis use. Human laboratory (Haney et al, 2013a) and clinical studies (Buckner et al, 2013) demonstrate that the negative affect associated with cannabis withdrawal (eg, irritability, anxiety, craving) can influence the return to cannabis use after abstinence. Cannabinoid type-1 (CB1) receptor agonists, such as dronabinol or nabilone, reduce cannabis withdrawal symptoms in both the laboratory and the clinic, and nabilone (but not dronabinol) reduced relapse to cannabis as measured in the human laboratory (Haney et al, 2004, 2013b; Budney et al, 2007; Vandrey et al, 2013; Levin et al, 2011). No clinical studies have tested whether CB1 agonists will prevent relapse in the clinic, that is, reduce the likelihood that abstinent cannabis smokers will return to 
cannabis use. Dronabinol did not reduce ongoing cannabis use in the clinic, yet most patients were not abstinent while taking dronabinol (Levin et al, 2011).

An alternative medication development strategy is to reduce ongoing drug use in individuals who are not abstinent by reducing the direct positive subjective (eg, cannabis 'liking' or 'good effect') and reinforcing effects (selfadministration) of the drug. The CB1 receptor mediates the positive subjective and reinforcing effects of cannabis (Cooper and Haney, 2008), and the subjective effects of cannabis are attenuated by the CB1 receptor antagonist, rimonabant (Huestis et al, 2001, 2007). Further study of rimonabant has, however, been discontinued following evidence that its chronic administration produced side effects such as depression and anxiety (Taylor, 2009), and the manufacturers of other CB1 receptor antagonists approved for testing in humans withdrew their clinical development after rimonabant use was suspended.

Opioid receptor antagonists offer an indirect approach to reducing the positive subjective and reinforcing effects of cannabis. There is a bidirectional modulatory relationship between the opioid and cannabinoid systems at the behavioral, neuroanatomical, and molecular levels (see, eg, Robledo et al, 2008; Scavone et al, 2013; Rios et al, 2006). For example, preclinical studies show that opioid antagonists reduce both the discriminative stimulus (Solinas and Goldberg, 2005; but see Wakley and Craft, 2011) and reinforcing effects of CB1 receptor agonists (Braida et al, 2001; Navarro et al, 2001; Justinova et al, 2004), suggesting that opioid receptor antagonists could reduce cannabis abuse liability. However, these effects have not been replicated in humans. In fact, in daily cannabis smokers, acute pretreatment with the opioid antagonist, naltrexone $(12-100 \mathrm{mg})$, increased the positive subjective effects of cannabis (Cooper and Haney, 2010).

It may be, however, that chronic naltrexone administration would produce different effects than acute pretreatment. A range of studies have shown that acute and chronic dopamine receptor antagonism can produce opposite effects on cocaine reinforcement in both human and non-human subjects (see Haney and Spealman, 2008), so the same may be true for opioid receptor antagonists and cannabis effects.

Given the clear interaction between opioid and cannabinoid drugs, the objective of this placebo-controlled, double-blind laboratory study was to assess the subjective, reinforcing, psychomotor, and physiological effects of active and inactive cannabis before, during, and after 16 days of daily naltrexone administration. We also measured naltrexone's effects on self-reported cannabis use outside the laboratory. Based on preclinical data, we hypothesized that maintenance on naltrexone would reduce both selfadministration and the positive subjective effects of cannabis, findings that would support its potential as a possible treatment for cannabis use disorder.

\section{MATERIALS AND METHODS}

\section{Participants}

Volunteers, aged 21-50 years, were recruited through newspaper advertisements. Those who met inclusion/exclusion criteria after an initial phone screen were invited to the laboratory for further screening. Before enrolling in the study, candidates provided a detailed drug and medical history, received medical and psychiatric evaluations, and signed consent forms detailing all aspects of the research. Participants were accepted into the study if they were healthy, as determined by physical examination, psychiatric screening, electrocardiogram, and urine and blood chemistries, were not regular users of drugs other than cannabis, nicotine, and caffeine, and were not seeking treatment for their cannabis use. Eligible participants smoked at least half a cannabis cigarette four or more times per week for the 4 weeks before screening, as determined by urine toxicology and self-report. Those who met the Diagnostic and Statistical Manual (of Mental Disorders), fourth edition, revised criteria for Axis 1 conditions that would benefit from medical intervention were also excluded. Females were excluded if they were pregnant or nursing. Current use of over-the-counter or prescription medication was also exclusionary.

Participants were told that the study objective was to determine the effects of cannabis in combination with FDA-approved medications in cannabis smokers. In order to maintain the study blind, they were told that they may receive an antidepressant (bupropion), a medication to treat muscle spasms (baclofen), a medication used to treat alcohol dependence (naltrexone), and/or a placebo. All procedures were approved by the New York State Psychiatric Institute Institutional Review Board and were in accordance with the Declaration of Helsinki.

\section{Study Design and Procedures}

The study included 10 outpatient sessions over the course of 4-6 weeks conducted at the New York State Psychiatric Institute. Participants were randomized to receive naltrexone or placebo for 16 consecutive days. Table 1 portrays a representative schedule of the study design for the naltrexone group. We tested the effects of active and inactive cannabis before naltrexone administration, after acute pretreatment, after 1 week and 2 weeks of daily naltrexone, and 1 week after termination of naltrexone administration. Both active and inactive cannabis were tested, so the specificity of naltrexone's effects to cannabinoid receptor activation could be observed.

Before study onset, participants received one to two practice sessions on the computerized tasks; no capsules or cannabis were administered in practice sessions. For experimental sessions, one capsule containing placebo or naltrexone (50 mg, ReVia, Dupont Pharma) was administered in size 00 opaque capsules with riboflavin $(50 \mathrm{mg})$ and lactose filler, prepared by the New York State Psychiatric Institute Research Pharmacy. Half of a cannabis cigarette $(0.0$ or $5.5 \% \mathrm{THC}$; ca. $800 \mathrm{mg}$ ) provided by the National Institute of Drug Abuse was smoked $45 \mathrm{~min}$ after naltrexone administration. A between-group design was used, with one randomly assigned group receiving naltrexone whereas the other received placebo. Both groups received both active and inactive cannabis weekly in counterbalanced order.

\section{Experimental Sessions}

Experimental sessions started at $0900 \mathrm{~h}$ and lasted for $\sim 7 \mathrm{~h}$. Participants were instructed to not eat breakfast before the 
Table I Representative Daily Dosing Schedule for the Naltrexone Group

\begin{tabular}{|c|c|c|c|c|c|c|c|}
\hline Day & $\mathbf{M}$ & Tu & $\mathbf{W}$ & Th & $\mathbf{F r}$ & Sa & Su \\
\hline Week 0 & & & I & 2 & 3 & 4 & 5 \\
\hline Naltrexone & $0 \mathrm{mg}$ & $0 \mathrm{mg}$ & $50 \mathrm{mg}$ & $50 \mathrm{mg}$ & $50 \mathrm{mg}$ & $50 \mathrm{mg}$ & $50 \mathrm{mg}$ \\
\hline Cannabis strength & $0 \%$ & $5.5 \%$ & $0 \%$ & $5.5 \%$ & - & - & - \\
\hline Lab visit/session no. & 1 & 2 & 3 & 4 & $\checkmark$ & - & - \\
\hline Week I & 6 & 7 & 8 & 9 & 10 & 11 & 12 \\
\hline Naltrexone & $50 \mathrm{mg}$ & $50 \mathrm{mg}$ & $50 \mathrm{mg}$ & $50 \mathrm{mg}$ & $50 \mathrm{mg}$ & $50 \mathrm{mg}$ & $50 \mathrm{mg}$ \\
\hline Cannabis strength & - & - & $0 \%$ & $5.5 \%$ & - & - & - \\
\hline Lab visit/session no. & $\checkmark$ & - & 5 & 6 & $\checkmark$ & & \\
\hline Week 2 & 13 & 14 & 15 & 16 & 17 & 18 & 19 \\
\hline Naltrexone & $50 \mathrm{mg}$ & $50 \mathrm{mg}$ & $50 \mathrm{mg}$ & $50 \mathrm{mg}$ & - & - & - \\
\hline Cannabis strength & - & - & $0 \%$ & $5.5 \%$ & - & - & - \\
\hline Lab visit/session no. & $\checkmark$ & - & 7 & 8 & - & - & - \\
\hline Post-NTX & 20 & 21 & 22 & 23 & & & \\
\hline Naltrexone & - & - & $0 \mathrm{mg}$ & $0 \mathrm{mg}$ & & & \\
\hline Cannabis strength & - & - & $0 \%$ & $5.5 \%$ & & & \\
\hline Lab visit/session no. & - & - & 9 & 10 & & & \\
\hline
\end{tabular}

Order of active and inactive cannabis administration within each 2-day block was counterbalanced. Capsule administration was observed by study staff in each session. Checkmarks indicate nonsession days in which participants came to the laboratory to take their capsules under observation. Plasma was drawn on days 6 and 13 to confirm daily naltrexone administration.

Table 2 Time Course of Sessions

\begin{tabular}{rlrl}
\hline Time & Event & Time & Event \\
\hline-90 & Begin session, TLFB & 120 & BP, VAS, CRF, MRF \\
& CO, Breathalyzer, Urine Toxicology & & Food available: ad libitum \\
& Balance & 150 & Choice: 0-3 cannabis puffs \\
& Light Breakfast & 165 & Cannabis self-administration \\
& BP, VAS, Task Battery & 180 & BP, VAS, CRF, MRF \\
-45 & Capsule administration & 195 & BP, VAS, CRF, MRF, Task Battery \\
-30 & CRF & 225 & BP, VAS, CRF, MRF \\
0 & Cannabis administration & 240 & Food options removed \\
15 & BP, VAS, CRF, MRF & 255 & BP, VAS, CRF, MRF, Task Battery \\
30 & BP, VAS, CRF, MRF, Task Battery & 285 & BP, VAS, CRF, MRF \\
60 & BP, VAS, CRF, MRF & 315 & BP, VAS \\
90 & BP, VAS, CRF, MRF, Task Battery & & Field Sobriety \\
& Performance Battery & & End session \\
& Immediate Word Recall & & \\
\hline
\end{tabular}

Abbreviations: $\mathrm{BP}$, blood pressure and heart rate; $\mathrm{CO}$, carbon monoxide test; CRF, capsule rating form; MRF, marijuana rating form; TLFB, timeline followback; VAS, visual analog scale of subjective effects;

Puffs cost $\$ 1.00$ each.

session and to refrain from using illicit drugs (other than cannabis, for which no instructions were given) for the duration of the study. Alcohol use was prohibited $24 \mathrm{~h}$ before and following each laboratory session, as was cannabis and tobacco use on the morning of sessions. A urine drug screen and a breath alcohol test were conducted at the beginning of each session to confirm compliance. Sessions were cancelled if there was evidence of illicit drug use, or alcohol or cannabis use on the morning of the session. If carbon monoxide levels indicated that the participant had smoked cannabis or tobacco before arrival ( $\geq 8$ p.p.m.), the session was rescheduled. In female participants, pregnancy tests were conducted at the beginning of the first and fourth session.

Table 2 portrays the schedule for experimental sessions. At the beginning of each session, we conducted a timeline followback questionnaire, querying about cannabis, alcohol, and other drug use, as well as any medication side effects since the last visit. Participants were then served a standardized breakfast. Following breakfast, baseline cardiovascular measures, balance (number of seconds the participants could balance for a maximum of $30 \mathrm{~s}$ on each foot; Evans et al, 1994), subjective-effects questionnaires, and performance tasks were completed, followed by naltrexone or placebo capsule administration. The participant and study staff were blind to capsule content. After capsule administration, half of a cannabis cigarette was smoked (see below). Cardiovascular and subjective-effects measures and a cognitive task battery were completed at baseline and at 30-60 min intervals following capsule and cannabis administration (see Table 2). Timing of each measurement was scheduled to capture the time course of naltrexone and cannabis effects. Food intake was measured for $2 \mathrm{~h}$, beginning $2 \mathrm{~h}$ after cannabis administration.

To measure the reinforcing effects of cannabis, $150 \mathrm{~min}$ after cannabis administration participants were offered the opportunity to purchase up to 3 additional 5-s puffs of the cannabis sampled that morning, with each puff costing $\$ 1$ of their study earnings. Cigarette smokers were permitted to smoke at predetermined intervals throughout the session in order to minimize nicotine withdrawal symptoms (maximum $=3$ tobacco cigarettes). At the end of each session, participants were free to leave after passing field sobriety and balancing tasks.

\section{Study Medications}

Naltrexone dose was randomized and cannabis strength was counterbalanced within each 2-day block. Cannabis (0.0, $5.5 \%$ THC), provided by the National Institute of Drug Abuse, was administered using a cued-smoking procedure (Foltin et al, 1987). Participants, viewed through a two-way mirror, were instructed through an intercom to 'light the cigarette' (30s), 'prepare' (5s), 'inhale' (5s), 'hold smoke in lungs' (10s), and 'exhale.' Participants smoked one puff every min, with a 40 -s interval between each puff, until they had smoked $50 \%$ of the cannabis cigarette. As the color of cannabis leaves varies as a function of THC content (Chait and Pierri, 1989), cigarettes were smoked through a cigarette holder and rolled at the end, so that the cannabis was not visible. Cannabis cigarettes were stored frozen in an airtight container and humidified at room temperature for $24 \mathrm{~h}$ before use.

Capsules, packed with riboflavin $(50 \mathrm{mg})$, were taken under observation of research staff on 10 of the 16 days of medication maintenance; participants either came into the laboratory to take their capsule under observation or they took the capsule during a laboratory session. When capsules were taken at home, compliance was assessed by observing urine under ultraviolet detection for riboflavin. Participants were also told that their blood could be drawn on any day to measure plasma medication levels, and that they would risk 
possible study discharge if they did not comply with instructions to take the medication at home. As shown in Table 1, we measured plasma naltrexone following the weekend in week 1 and week 2. Participants in the placebo group had their blood drawn to maintain the blind, but no assays were conducted.

\section{Assessments}

Subjective mood and drug effects. All subjective effects were measured using visual analog scales (VAS), a series of $100 \mathrm{~mm}$ long lines labeled 'not at all' at one end $(0 \mathrm{~mm})$ and 'extremely' at the other end $(100 \mathrm{~mm})$. Participants were instructed to rate their subjective experiences according to how they felt at that moment. Measurements were taken at baseline and 5 times from 15 to $120 \mathrm{~min}$ after cannabis administration. The mean of post-baseline measurements was analyzed.

Symptom scale. Participants completed a 44-item scale assessing a range of affective and physical symptoms capturing effects associated with both cannabis (eg, 'mellow') and naltrexone (eg, stomach upset; Haney et al, 1999).

Marijuana rating form (MRF). Subjective cannabisrelated drug effects were assessed using a 5-item VAS asking participants to rate the strength of the cannabis effect, good effect, bad effect, drug liking, and willingness to smoke the marijuana again. Measurements were taken at baseline and 5 times from 15 to $120 \mathrm{~min}$ after cannabis administration. The mean of post-baseline measurements was used in the analysis.

Capsule rating form (CRF). Participants completed a 5-item VAS, rating the strength of the drug effect, good effect, bad effect, willingness to take drug again, and drug liking. In addition, participants were asked to indicate whether they thought the drug was most like a placebo, sedative, or stimulant. Measurements were taken at 11 times from 15 to $330 \mathrm{~min}$ after capsule administration. The majority of values were 0 , and a dichotomous measurement $(0 v s>0)$ was used in the analysis.

Food intake. Participants received a box of food containing a variety of meal items (eg, tuna, turkey, soup), snacks (eg, cookies, fruit, ice cream, candy), and beverages (eg, soda, juice, iced tea) that could be consumed ad libitum over a 2-h period, starting $2 \mathrm{~h}$ after cannabis administration. Frozen meal items (eg, meat loaf, pasta, pizza) were also available by request during this time. Additional units of any item were available. Participants recorded the time and the portion size of any item consumed, under the observation of research assistants. Staff verified food consumption by sorting food trash at the end of the session. Caloric and macronutrient content of each item consumed was analyzed.

Cognition. Cognitive function was assessed with a computerized battery including: a Digit Span immediate and delayed recall and recognition task (DIG; $2 \mathrm{~min}$ ); a Divided Attention Task (DAT; 10 min); a Digit-Symbol Substitution Task (DSST; 3 min); and a Repeated Acquisition Task (RAT; $3 \mathrm{~min}$ ). This battery, designed for repeated assessments, measures basic as well as higher-level cognitive functions including psychomotor speed, simple and divided attention, and verbal and working memory. We have previously shown this battery to be sensitive to the effects of cannabinoids (Bedi et al, 2010; Hart et al, 2005). Measurements were taken at baseline and at 30 and 90 min after cannabis administration. All tasks generated data that were continuous except for the DIG that was analyzed as a dichotomous measure.

Cardiovascular function. Heart rate and seated blood pressure were measured with a Sentry II vital signs monitor (Model 6100: NBS Medical Services, Costa Mesa, CA). Measurements were taken at baseline and 5 times between 15 and $120 \mathrm{~min}$ after cannabis administration. The mean of post-baseline measurements was analyzed.

Plasma naltrexone measures. Naltrexone and its metabolite, $6 \beta$-naltrexol, were quantified after liquid/liquid extraction and derivitization with heptafluorobenzoyl chloride using Capillary GC/Mass spectrometry operated in the simultaneous Ion monitoring mode with deuterated internal standards. Intra- and inter-assay coefficients of variation were $<6$ and $8 \%$ respectively. The assay measured unconjugated analyte.

Data analysis. The continuous outcomes, subjectiveeffects ratings (VAS, MRF), cognitive task performance (DAT: track distance; DSST: total correct; RAT: total entered), food intake, and cardiovascular data, were modeled with longitudinal linear mixed effects models as a function of naltrexone dose, time (weeks 0,1 , and 2 , and 1 week after naltrexone), naltrexone by time interaction, and baseline data (outcome measurement during inactive and active marijuana) before naltrexone randomization; and a baseline measurement of the outcome at each timepoint, when available, using random intercept and autoregressive (AR1) correlation structure to account for within-subject correlation. If the naltrexone dose by time interaction was found significant, between-group contrasts were tested at each timepoint, and significant contrasts were reported. If the naltrexone dose by time interaction was not significant, a model with the main effects of naltrexone dose, time, and baseline data was fit as the final model. The dichotomous outcomes, self-administration, CRF, and cognitive task performance (DIG: total correct), were modeled similarly, but utilized longitudinal logistic mixed effects models. Note, only subjective-effects, cognitive, and cardiovascular data collected during the $2 \mathrm{~h}$ following the morning administration of marijuana were analyzed; in the afternoon, these data were confounded by variations between participants in selfadministered cannabis. Furthermore, in order to reduce the number of analyses made, we only analyzed 6 of the 44 -item VAS items considered most relevant to cannabis' positive subjective effects: Good Effect, High, Mellow, Marijuana craving, Cigarette craving, and Friendly.

We analyzed timeline followback data to answer two questions. (1) Did the number of cannabis cigarettes smoked per day change over time between treatment groups, modeled longitudinally as a function of treatment, day, two-way day by treatment interaction, and baseline marijuana use? (2) Did the day in which cannabis use peaked 
differ as function of treatment, using a Wilcoxon rank-sum test (nonparametric version of two-sample $t$-test) and a Kolmogorov-Smirnov Test (nonparametric test comparing distributions) in order to determine whether naltrexone influenced patterns of cannabis use outside of the laboratory? We also analyzed timeline followback alcohol data for those who were alcohol users, and dichotomized the outcome to either using or not using. The dichotomous drinking outcome was modeled with a longitudinal logistic mixed effects model as a function of treatment, day, day by treatment interaction, and baseline alcohol use. The interaction term was removed if not found significant.

\section{RESULTS}

Table 3 describes the demographic information of the research volunteers receiving placebo $(n=26 \mathrm{M}$ and $2 \mathrm{~F})$

Table 3 Demographic Characteristics of Participants

\begin{tabular}{lcc}
\hline & Placebo & Naltrexone \\
\hline Number of participants & $28(26 \mathrm{M}$ and $2 \mathrm{~F})$ & $23(18 \mathrm{M}$ and $5 \mathrm{~F})$ \\
Race (BlackMhite/Mixed) & $22 / 4 / 2$ & $15 / 7 / 1$ \\
Ethnicity (Hispanic/non-Hispanic) & $3 / 25$ & $5 / 18$ \\
Age (years) & $31.6 \pm 8.0$ & $28.6 \pm 7.0$ \\
Cannabis use (no. of days/week) & $6.3 \pm 1.0$ & $6.4 \pm 1.0$ \\
Cannabis cigarettes/day & $6.0 \pm 4.0$ & $5.5 \pm 3.6$ \\
Years daily cannabis use & $12.7 \pm 7.9$ & $11.1 \pm 8.6$ \\
Alcohol drinkers ${ }^{\mathrm{a}}$ (\%) & 46 & 43 \\
Alcohol drinks/week & $8.6 \pm 10.8$ & $12.3 \pm 15.7$ \\
Education (years) & $12.5 \pm 1.6$ & $12.4 \pm 1.6$ \\
\hline
\end{tabular}

Data are presented as means $( \pm S D)$ or as frequency.

${ }^{a}$ Alcohol drinkers are defined as those reporting at least one drink per week. Participants reported no current drug use other than cannabis and possibly

tobacco and alcohol. or naltrexone ( $n=18 \mathrm{M}$ and $5 \mathrm{~F}$ ) who completed the study. Demographic variables were comparable across the two groups, as was the number of participants who failed to complete the study (placebo: $n=8$; naltrexone: $n=9$ ). Reasons for discontinuation were: not adhering to the protocol $(n=12)$, not liking capsule effects $(n=3)$, or an adverse event after smoking active cannabis $(n=2$; panic attack, loss of consciousness).

\section{Cannabis Self-Administration}

Because at least $50 \%$ of participants chose to not selfadminister any active cannabis across the 10 sessions, the number of cannabis puffs self-administered was dichotomized (no puffs, $\geq 1$ puff), and analyzed using longitudinal logistic mixed effects models. Figure 1, portraying the percentage of participants self-administering inactive and active cannabis, shows that self-administration of inactive cannabis was low and not significantly influenced by naltrexone (left figure). However, naltrexone significantly decreased self-administration of active cannabis relative to placebo $(\mathrm{F}(1,150)=4.33, p=0.04$; right figure $)$. Participants in the placebo group had 7.6 times (95\% CI: $1.1-51.8)$ the odds of self-administering active cannabis compared with the naltrexone group; the interaction between naltrexone and time was not significant for self-administration or any other measure tested unless noted, and hence the interaction has been removed from the final models.

\section{Cannabis Rating and Visual Analog Scales}

Figure 2, portraying ratings of 'Good Effect' as a function of cannabis strength, naltrexone dose, and time, shows that ratings were low and unaltered by naltrexone following inactive cannabis administration (left figure). Under conditions of active cannabis administration (right figure), the naltrexone group had significantly lower ratings of 'Good Effect' compared with the placebo group, when controlling for

Self-Administration

Inactive Cannabis
Active Cannabis*

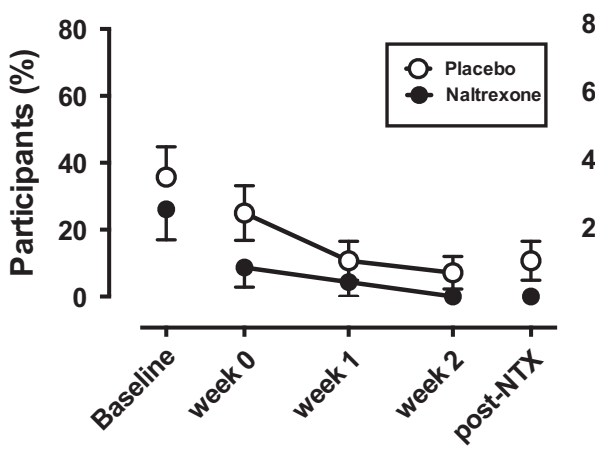

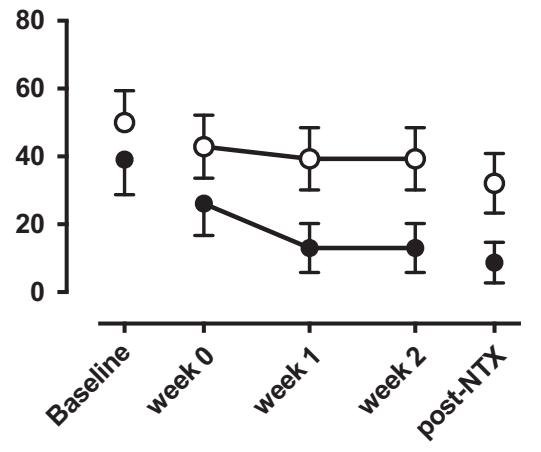

Time

Figure I Percentage of participants self-administering inactive $(0.0 \% \mathrm{THC})$ or active $(5.5 \% \mathrm{THC})$ cannabis, that is, purchasing at least I puff of cannabis available for self-administration ( $\max =3$ puffs) as a function of time and naltrexone dose. Baseline refers to the session before naltrexone administration commenced. Post-NTX refers to the session that occurred at least I week following termination of naltrexone administration. All participants received placebo capsules during baseline and post-NTX sessions. *P<0.05, significant difference between the naltrexone and placebo groups. Error bars represent \pm SEM. 


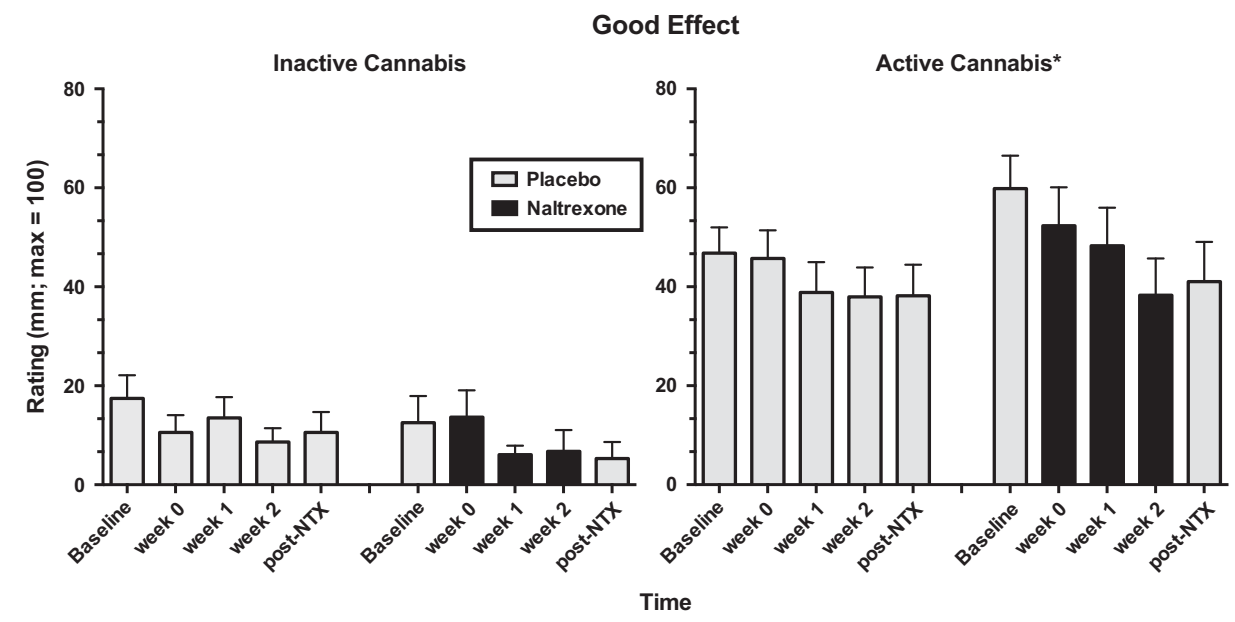

Figure 2 Mean effects of inactive (0.0\% THC) and active cannabis (5.5\% THC) on ratings of 'Good Effect' as a function of naltrexone condition and time. Note the data were statistically analyzed including baseline measures as a covariate. Data collected for $2 \mathrm{~h}$ after cannabis administration were averaged. See Figure I for details.

Timeline Followback: Day of Peak Cannabis Use

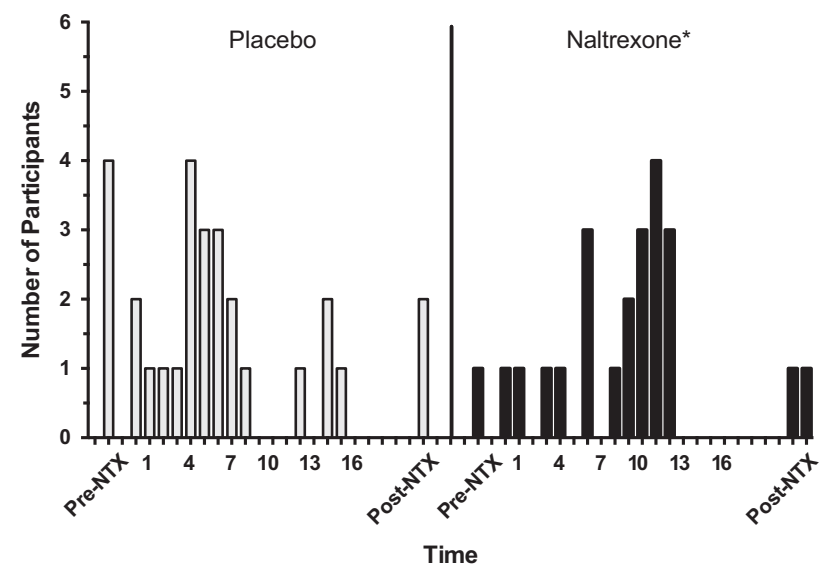

Figure 3 Number of participants reporting peak cannabis use in their natural environment as a function of time. Participants were asked to describe the amount of cannabis smoked since their last laboratory visit. PreNTX refers to cannabis smoked outside the laboratory on the day before the first session and on the two baseline sessions. Days $1-15$ are portrayed; data were not collected for days 16-20. Post-NTX refers to cannabis smoked outside the laboratory on the days 21 and 22; data were not collected for day 23. $* P<0.05$, significant difference between the distribution of peak cannabis use in the naltrexone and placebo groups.

differences in baseline $(\mathrm{F}(1,149)=4.54, p=0.03)$. Naltrexone did not significantly affect other subjective-effects ratings such as cannabis craving or 'High' in either cannabis condition, but did produce significantly lower ratings of 'Friendly' following both active $(\mathrm{F}(1,148)=4.06, p=0.046)$ and inactive cannabis administration (Figure 3; $\mathrm{F}(1,149)=4.63, p=0.03$ ) over the course of the study (data not shown).

\section{Capsule Rating Form}

This visual analog data set had a large number of zeros (scale $0-100 \mathrm{~mm})$, and hence the data were dichotomized $(0,>0)$.
Naltrexone only significantly altered one capsule rating, 'liking.' Under conditions of inactive cannabis administration, there was a significant main effect of naltrexone: participants in the placebo group had significantly higher odds of liking the capsules (ie, ratings $>0$ ) compared with those in the naltrexone group (OR $=22.5$ (95\% CI: 1.6-319.9), $\mathrm{F}(1,145)=5.30, p=0.02)$. Under conditions of active cannabis administration, there was a significant naltrexone by time interaction for ratings of capsule liking $(F(3,145)=5.20, p=0.002)$. Participants in the placebo group had significantly higher odds of liking the capsules (ie, ratings $>0$ ) compared with participants in the naltrexone group at week 1 (OR $=46.2$ (95\% CI: 3.3-647.6), $t_{145}=2.84$, $p=0.005)$ and week $2(\mathrm{OR}=24.6(95 \% \mathrm{CI}: 1.7-365.2)$, $t_{145}=2.33, p=0.02$; data not shown).

\section{Food Intake}

Following inactive cannabis administration, the naltrexone group had significantly lower caloric intake during ad libitum food availability (measured for $2-4 \mathrm{~h}$ after cannabis smoking) than the placebo group $(\mathrm{F}(1,146)=4.32, p=0.04)$. For example, by week 3, caloric intake per session in the placebo group was $1310 \pm 200 \mathrm{kcals}$ compared with $850 \pm 200$ kcals in the naltrexone group. Naltrexone reduced caloric intake without altering the proportion of calories derived from fat, carbohydrates, or protein. The naltrexone group also had lower food intake following active cannabis smoking compared with the placebo group $(\mathrm{F}(1$, $148)=18.66, p<0.0001$ ), but these data are confounded by self-administration: as shown in Table 2, food intake was measured before and after cannabis was available for selfadministration, and hence naltrexone's effects on cannabis self-administration could have contributed to a decrease in food intake.

\section{Cognitive End Points}

Naltrexone did not significantly influence the effect of either inactive or active cannabis' effects on cognitive task 
performance relative to placebo, except that there was a significant time by naltrexone condition interaction $(\mathrm{F}(3$, 144) $=3.50, p=0.02$ ) for the Digit Recall Task: following inactive cannabis administration, participants in the naltrexone group had higher odds of entering at least 8 digit strings in the Immediate Recall phase of this task as compared with those in the placebo group in the first study week $(\mathrm{OR}=7.9$ (95\% CI: $1.7-37.0), t_{144}=2.64, p=0.009$ ).

\section{Cardiovascular End Points}

Following inactive cannabis administration, the naltrexone group had a small but significant decrease $(\sim 3 \mathrm{~mm} \mathrm{Hg})$ in systolic blood pressure compared with the placebo group $(\mathrm{F}(1,149)=4.45, p=0.04)$. Naltrexone did not significantly influence heart rate or blood pressure following active cannabis administration.

\section{Timeline Followback}

The number of cannabis cigarettes smoked per day outside of the laboratory, ranging between 0.38 and 9.70 per day over the course of the study, did not show a significant two-way interaction between day and treatment. However, the day each participant reported the most cannabis use outside of the laboratory, portrayed as a histogram in Figure 3, significantly differed in the placebo and naltrexone group (median: placebo group $=5$, naltrexone group $=11, U=703.5$, $p=0.046)$. The Kolmogorov-Smirnov test demonstrated a significant difference in frequency distribution between the two groups $(\mathrm{D}=0.40, p=0.03)$. The placebo group showed a more distributed pattern of peak cannabis use over time, whereas peak cannabis use in the naltrexone group clustered toward the last week of daily capsule administration.

Given naltrexone's effects on alcohol use, we were also interested in assessing the number of alcohol drinks consumed outside the laboratory as a function of treatment condition. However, less than half the participants in either group consumed any alcohol (Table 3). Among those who did, the use was low (mean: 9-10 drinks/week during the study), with the majority of days (58.7\%) being alcohol free. Therefore, the drinking outcome was dichotomized. In the final main effects model, the odds of drinking did not significantly differ between treatment groups. No other drug use was reported in the TLFB.

\section{Medication Side Effects}

Table 4 shows the number of times medication side effects were reported over 16 days of medication administration. The primary adverse events reported were gastrointestinal (GI) upset (nausea, stomach cramps, vomiting), headache, jitteriness, and fatigue; all but fatigue occurred more frequently in the naltrexone group than the placebo group. The placebo group reported more muscle ache and urination frequency. In the naltrexone group, $83 \%$ of participants reported at least one side effect, compared with $57 \%$ of the placebo group $\left(\chi_{1}^{2}=3.80, p=0.051\right)$.
Table 4 Medication Side Effects

\begin{tabular}{lcc}
\hline & Placebo & Naltrexone \\
\hline Gastrointestinal upset & 16 & 76 \\
Headache & 8 & 24 \\
Jittery/restless & 9 & 23 \\
Fatigue & 25 & 30 \\
Muscle ache & 9 & 4 \\
Lightheaded/dizzy & 2 & 7 \\
Increased urination & 10 & 0 \\
Increased heart Rate & 2 & 7 \\
\hline
\end{tabular}

Frequency of each symptom was reported over the 16 days of daily medication administration (symptoms occurring $>6$ times are portrayed). In the naltrexone group, $83 \%$ of participants reported at least one side effect as compared with $57 \%$ of the placebo group $\left(\chi_{1}^{2}=3.80, p=0.051\right)$.

\section{Plasma Naltrexone}

Plasma naltrexone levels were measured both during week 1 (day 8) and week 2 (day 15) of capsule administration to confirm medication compliance when capsules were not administered under observation. Plasma levels of unconjugated naltrexone and $6 \beta$-naltrexone averaged (mean: SD) $1.1 \pm 1.9$ and $15.8 \pm 10.9 \mathrm{ng} / \mathrm{ml}$, respectively.

\section{DISCUSSION}

This human laboratory study shows that maintenance on naltrexone significantly decreased the reinforcing effects of active cannabis relative to placebo capsule administration. After at least 1 week of naltrexone maintenance, the number of participants self-administering active cannabis was as low as the number self-administering inactive cannabis. Naltrexone also decreased ratings of 'good effect' following active cannabis smoking.

This attenuation of reinforcing and positive subjective effects appeared to have influenced cannabis use in the natural ecology. Although the average number of marijuana cigarettes that participants smoked outside of the laboratory did not significantly vary as a function of time or naltrexone dose, we analyzed the day individual participants smoked the most cannabis during their study participation, and observed that cannabis use peaked at about the time that naltrexone was attenuating cannabis' effects in the laboratory. This suggests that participants, who were explicitly not interested in reducing their cannabis use, may have smoked more cannabis in their natural environment to overcome naltrexone's effects. In the laboratory, surmounting a blunted effect was not possible, as only three puffs of cannabis were available for self-administration.

Whether patients in treatment would reduce their use in response to an attenuated cannabis effect is an empirical question, but this is the mechanism by which naltrexone reduces alcohol use clinically (see, eg, Anton et al, 2006). Naltrexone reduced alcohol self-administration in alcoholdependent, nontreatment-seeking research volunteers (O’Malley et al, 2002), similar to the present study, and also decreased the likelihood that alcohol-dependent patients who lapsed after a period of abstinence would return to heavy drinking (O’Malley et al, 1992; Volpicelli et al, 1992). 
Reducing drug use may, in fact, be a particularly relevant objective for cannabis treatment. Although abstinence is the goal of most treatment programs, the goal of many patients is to moderate their cannabis use rather than quit altogether (Lozano et al, 2006). As with alcohol, naltrexone may reduce ongoing heavy cannabis use, relapse severity, or the likelihood that patients would return to pretreatment levels of heavy cannabis use in the event of a lapse.

These findings are largely consistent with preclinical data showing that opioid receptor antagonists reduce cannabinoid agonist self-administration (Braida et al, 2001; Navarro et al, 2001; Justinova et al, 2004). Our earlier findings in cannabis smokers showing that acute naltrexone administration (eg, days 1 and 2 in the present design) enhanced cannabis intoxication were not replicated (Cooper and Haney, 2010). One possible explanation for this lack of replication may involve cannabis strength: the earlier study tested less potent cannabis (3.27\% THC), producing lower ratings of 'High' and 'Good Effect' than the higher strength cannabis tested herein (5.5\%). The relationship between naltrexone and cannabinoids is complex and appears to be influenced by a variety of experimental parameters including route of administration (oral THC vs smoked cannabis), THC dose, naltrexone dose, outcome measure (reinforcement $v s$ analgesia), and cannabis use patterns (daily vs infrequent; Haney, 2007; Haney et al, 2003; Greenwald and Stitzer, 2000; Wachtel and de Wit, 2000). The underlying mechanism contributing to the variable effects observed across experimental parameters remains unknown.

In terms of other behavioral outcomes, naltrexone improved performance on one cognitive task following active relative to placebo cannabis administration. Naltrexone also decreased food intake, a well-known effect (see, eg, Lee and Fujioka, 2009) that occurred under both active and inactive cannabis conditions. Naltrexone's other intrinsic effects include decreasing ratings of 'Friendly' and capsule liking and decreasing systolic blood pressure. In addition, participants maintained on naltrexone reported more frequent GI upset and headaches relative to the placebo group, symptoms that are also reported by patients treated with naltrexone for alcohol dependence (see Anton, 2008). Importantly, participants in the naltrexone group did not drop out of the study more than the placebo group, suggesting that the side effects were not severe enough to affect study completion.

Compliance in taking the medications was high under these carefully controlled laboratory conditions, as confirmed by qualitative riboflavin assessments and plasma medication levels: trough levels of $6 \beta$-naltrexone (ie, $\sim 24 \mathrm{~h}$ after the last naltrexone administration) closely paralleled those reported in studies with confirmed medication administration (Mason et al, 2002; Verebey et al, 1976). Compliance was prioritized in this laboratory study, with a level of oversight that would be difficult to maintain in clinical studies. Yet, naltrexone's side effects could affect study completion and compliance (see, eg, Oncken et al, 2001; Pettinati et al, 2000), and hence strategies to ensure compliance would need to be prioritized if naltrexone is tested clinically for CUD. Trials with depot formulations of naltrexone, obviating concerns of daily medication administration, may be particularly appropriate.

To summarize, given the widespread and increasing use of cannabis, the number of individuals developing CUD will only increase, furthering the need for pharmacotherapeutic treatment options. As with drugs such as alcohol (O'Malley et al, 1992; Volpicelli et al, 1992), opioids (Comer et al, 2006; Krupitsky et al, 2011), and amphetamines (JayaramLindström et al, 2008), naltrexone maintenance reduced the direct reinforcing and positive subjective effects of smoked cannabis. Of note, of the 11 medications tested in our human laboratory as potential treatments for CUD, few have reduced cannabis self-administration, even those exerting important effects, like improving sleep during cannabis withdrawal (see Balter et al, 2014). Given that a medication's effects on drug self-administration in the laboratory is, to date, the best predictor of its effects on drug-taking among patients in the clinic (Haney and Spealman, 2008; Comer et al, 2008; Haney, 2009; Stoops and Rush, 2013), clinical studies testing the effects of naltrexone maintenance, with careful consideration of medication compliance, are warranted to determine naltrexone's efficacy as a treatment of cannabis dependence.

\section{FUNDING AND DISCLOSURE}

Dr Haney's research is funded by NIDA. She has received partial salary support for investigator-initiated studies from Astra-Zeneca, Insys Therapeutics, and Lifeloc Technologies, and has served as a consultant to Aelis Farma and Health Advances LLC. Dr Cooper is on the scientific advisory board of KannaLife Sciences. The other authors declare no conflict of interest.

\section{ACKNOWLEDGMENTS}

This research was supported by the US National Institute on Drug Abuse (NIDA: DA19239 and DA09236). We are grateful to Dr Richard W Foltin for his support in conducting this study and thank Dr Adam Bisaga for his medical supervision.

\section{REFERENCES}

Anton RF (2008). Naltrexone for the management of alcohol dependence. $N$ Engl J Med 359: 715-721.

Anton RF, O'Malley SS, Ciraulo DA, Cisler RA, Couper D, Donovan DM et al (2006). Combined pharmacotherapies and behavioral interventions for alcohol dependence: the COMBINE study: a randomized controlled trial. JAMA 295: 2003-2017.

Balter R, Cooper ZD, Haney M (2014). Novel pharmacologic approaches to treating cannabis use disorder (invited review). Curr Addict Rep 1: 137-143.

Bedi G, Foltin RW, Gunderson EW, Rabkin J, Hart CL, Comer SD et al (2010). Efficacy and tolerability of high-dose dronabinol maintenance in HIV-positive marijuana smokers: a controlled laboratory study. Psychopharmacology 212: 675-686.

Braida D, Pozzi M, Cavallini R, Sala M (2001). Conditioned place preference induced by the cannabinoid agonist CP 55,940: interaction with the opioid system. Neuroscience 104: 923-926.

Buckner JD, Zvolensky MJ, Ecker AH (2013). Cannabis use during a voluntary quit attempt: an analysis from ecological momentary assessment. Drug Alcohol Depend 132: 610-616.

Budney AJ, Moore BA, Rocha HL, Higgins ST (2006). Clinical trial of abstinence-based vouchers and cognitive-behavioral therapy for cannabis dependence. J Consult Clin Psychol 74: 307-316. 
Budney AJ, Vandrey RG, Hughes JR, Moore BA, Bahrenburg B (2007). Oral delta-9-tetrahydrocannabinol suppresses cannabis withdrawal symptoms. Drug Alcohol Depend 86: 22-29.

Cerdá M, Wall M, Keyes KM, Galea S, Hasin D (2012). Medical marijuana laws in 50 states: investigating the relationship between state legalization of medical marijuana and marijuana use, abuse and dependence. Drug Alcohol Depend 120: 22-27.

Chait LD, Pierri J (1989). Some physical characteristics of NIDA marijuana cigarettes. Addict Behav 14: 61-67.

Comer SD, Sullivan MA, Yu E, Rothenberg JL, Kleber HD, Kampman K et al (2006). Injectable, sustained-release naltrexone for the treatment of opioid dependence: a randomized, placebocontrolled trial. Arch Gen Psychiatry 63: 210-218.

Comer SD, Ashworth JB, Foltin RW, Johanson CE, Zacny JP, Walsh SL (2008). The role of human drug self-administration procedures in the development of medications. Drug Alcohol Depend 96: 1-15.

Cooper ZD, Haney M (2008). Cannabis reinforcement and dependence: role of the cannabinoid CB1 receptor. Addict Biol 13: $188-195$

Cooper ZD, Haney M (2010). Opioid antagonism enhances marijuana's effects in heavy marijuana smokers. Psychopharmacology 211: 141-148.

Evans SM, Troisi JR 2nd, Griffiths RR (1994). Tandospirone and alprazolam: comparison of behavioral effects and abuse liability in humans. J Pharmacol Exp Ther 271: 683-694.

Foltin RW, Brady JV, Fischman MW, Emurian CS, Dominitz J (1987). Effects of smoked marijuana on social interaction in small groups. Drug Alcohol Depend 20: 87-93.

Greenwald MK, Stitzer ML (2000). Antinociceptive, subjective and behavioral effects of smoked marijuana in humans. Drug Alcohol Depend 59: 261-275.

Haney M (2007). Opioid antagonism of cannabinoid effects: differences between marijuana smokers and nonmarijuana smokers. Neuropsychopharmacology 32: 1391-1403.

Haney M (2009). Self-administration of cocaine, cannabis and heroin in the human laboratory: benefits and pitfalls. Addict Biol 14: 9-21.

Haney M, Bedi G, Cooper ZD, Glass A, Vosburg SK, Comer SD et al (2013a). Predictors of marijuana relapse in the human laboratory: robust impact of tobacco cigarette smoking status. Biol Psychiatry 73: $242-248$

Haney M, Cooper ZD, Bedi G, Vosburg SK, Comer SD, Foltin RW (2013b). Nabilone decreases marijuana withdrawal and a laboratory measure of marijuana relapse. Neuropsychopharmacology 38: 1557-1565.

Haney M, Bisaga A, Foltin RW (2003). Interaction between naltrexone and oral THC in heavy marijuana smokers. Psychopharmacology 166: 77-85.

Haney M, Hart CL, Vosburg SK, Nasser J, Bennett A, Zubaran C et al (2004). Marijuana withdrawal in humans: effects of oral THC or divalproex. Neuropsychopharmacology 29: 158-170.

Haney M, Spealman R (2008). Controversies in translational research: drug self-administration. Psychopharmacology (Berl) 199: 403-419.

Haney M, Ward AS, Comer SD, Foltin RW, Fischman MW (1999). Abstinence symptoms following smoked marijuana in humans. Psychopharmacology 141: 395-404.

Hart CL, Haney M, Vosburg SK, Comer SD, Foltin RW (2005). Reinforcing effects of oral Delta9-THC in male marijuana smokers in a laboratory choice procedure. Psychopharmacology 181: 237-243.

Huestis MA, Boyd SJ, Heishman SJ, Preston KL, Bonnet D, Le Fur G et al (2007). Single and multiple doses of rimonabant antagonize acute effects of smoked cannabis in male cannabis users. Psychopharmacology 194: 505-515.

Huestis MA, Gorelick DA, Heishman SJ, Preston KL, Nelson RA, Moolchan ET et al (2001). Blockade of effects of smoked marijuana by the CB1-selective cannabinoid receptor antagonist SR141716. Arch Gen Psychiatry 58: 322-328.

Jayaram-Lindström N, Hammarberg A, Beck O, Franck J (2008). Naltrexone for the treatment of amphetamine dependence: a randomized, placebo-controlled trial. Am J Psychiatry 165: $1442-1448$.

Justinova Z, Tanda G, Munzar P, Goldberg SR (2004). The opioid antagonist naltrexone reduces the reinforcing effects of Delta 9 tetrahydrocannabinol (THC) in squirrel monkeys. Psychopharmacology 173: 186-194.

Kadden RM, Litt MD, Kabela-Cormier E, Petry NM (2007). Abstinence rates following behavioral treatments for marijuana dependence. Addict Behav 32: 1220-1236.

Krupitsky E, Nunes EV, Ling W, Illeperuma A, Gastfriend DR, Silverman BL (2011). Injectable extended-release naltrexone for opioid dependence: a double-blind, placebo-controlled, multicentre randomised trial. Lancet 377: 1506-1513.

Lee MW, Fujioka K (2009). Naltrexone for the treatment of obesity: review and update. Exp Opin Pharmacother 10: 1841-1845.

Levin FR, Mariani JJ, Brooks DJ, Cheng W, Nunes EV (2011). Dronabinol for the treatment of cannabis dependence: a randomized, double-blind, placebo-controlled trial. Drug Alcohol Depend 116: 142-150.

Lozano BE, Stephens RS, Roffman RA (2006). Abstinence and moderate use goals in the treatment of marijuana dependence. Addiction 101: 1589-1597.

Marijuana Treatment Project Research Group (2004). Brief treatments for cannabis dependence: findings from a randomized multisite trial. J Consult Clin Psychol 72: 455-466.

Mason BJ, Goodman AM, Dixon RM, Abdel Hameed MH, Hulot T, Wesnes $\mathrm{K}$ et al (2002). A pharmacokinetic and pharmacodynamic drug interaction study of acamprosate and naltrexone. Neuropsychopharmacology 27: 596-606.

Navarro M, Carrera MR, Fratta W, Valverde O, Cossu G, Fattore L et al (2001). Functional interaction between opioid and cannabinoid receptors in drug self-administration. J Neurosci 21: 5344-5350.

O'Malley SS, Jaffe AJ, Chang G, Schottenfield RS, Meyer RE, Rounsaville B (1992). Naltrexone and coping skills therapy for alcohol dependence. A controlled study. Arch Gen Psychiatry 49: 881-887.

O'Malley SS, Krishnan-Sarin S, Farren C, Sinha R, Kreek MJ (2002). Naltrexone decreases craivng and alcohol self-administration in alcohol-dependent subjects and activates the hypothalamopituitary-adrenocortical axis. Psychopharmacology 160: 19-29.

Oncken C, Van Kirk J, Kranzler HR (2001). Adverse effects of oral naltrexone: analysis of data from two clinical trials. Psychopharmacology 154: 397-402.

Pettinati HM, Volpicelli JR, Pierce JD Jr, O'Brien CP (2000). Improving naltrexone response. $J$ Addict Dis 19: 71-83.

Rios C, Gomes I, Devi LA (2006). Mu opioid and CB1 cannabinoid receptor interactions: Reciprocal inhibition of receptor signaling and neuritogenesis. Br J Pharmacol 148: 387-395.

Robledo P, Berrendero F, Ozaita A, Maldonado R (2008). Advances in the field of cannabinoid-opioid cross-talk. Addict Biol 13: 213-224.

Scavone JL, Sterling RC, Van Bockstaele EJ (2013). Cannabinoid and opioid interactions: implications for opiate dependence and withdrawal. Neuroscience 248: 637-654.

Solinas M, Goldberg SR (2005). Involvement of mu-, delta- and kappa-opoioid receptor subtypes in the discriminative-stimulus effects of delta-9-tetrahydrocannabinol (THC) in rats. Psychopharmacology 179: 804-812.

Stoops WW, Rush CR (2013). Agonist replacement for stimulant dependence: a review of clinical research. Curr Pharm Des 19: 7026-7035.

Substance Abuse and Mental Health Services Administration (SAMHSA), Center for Behavioral Health Statistics and Quality (2012). Drug Abuse Warning Network (DAWN), 2008: National Estimates of Drug-Related Emergency Department VisitsHHS Publication No. (SMA) 11-4618. SAMHSA: Rockville, MD. 
Substance Abuse and Mental Health Services Administration (SAMHSA) (2013). Results from the 2012 National Survey on Drug Use and Health: summary of national findingsNSDUH Series H-46, HHS Publication No. (SMA) 13-4795. SAMHSA: Rockville, MD.

Taylor D (2009). Withdrawal of rimonabant - walking the tightrope of 21st centry pharmaceutical regulation? (Opinion). Curr Drug Safety 4: 2-4.

Vandrey R, Stitzer ML, Mintzer MZ, Huestis MA, Murray JA, Lee D (2013). The dose effects of short-term dronabinol (oral THC) maintenance in daily cannabis users. Drug Alcohol Depend 128: 64-70.

Verebey K, Volavka J, Mule SJ et al (1976). Naltrexone: disposition, metabolism, and effects after acute and chronic dosing. Clin Pharmacol Ther 20: 315-328.
Volpicelli JR, Alterman AI, Hayashida M, O'Brien CP (1992). Naltrexone in the treatment of alcohol dependence. Arch Gen Psychiatry 49: 876-880.

Wachtel SR, de Wit H (2000). Naltrexone does not block the subjective effects of oral Delta(9)-tetrahydrocannabinol in humans. Drug Alcohol Depend 59: 251-260.

Wakley AA, Craft RM (2011). THC-methadone and THCnaltrexone interactions on discrimination, antinociception, and locomotion in rats. Behav Pharmacol 22: 489-497.

Weinstein AM, Miller H, Bluvstein I, Rapoport E, Schreiber S, Bar-Hamburger $\mathrm{R}$ et al (2014). Treatment of cannabis dependence using escitalopram in combination with cognitive-behavior therapy: a double-blind placebo-controlled study. Am J Drug Alcohol Abuse 40: 16-22. 